\title{
Effects of spatial cuing on the onset repulsion effect
}

\author{
Timothy L. Hubbard • Susan E. Ruppel
}

Published online: 20 July 2011

(C) Psychonomic Society, Inc. 2011

\begin{abstract}
Effects of cuing the onset (initial) location of a moving target on memory for the onset location of that target were examined. If a cue presented prior to target onset indicated the location where that target would appear, the onset repulsion effect (in which the judged initial location of the target was displaced in the direction opposite to target motion) was decreased, and the onset repulsion effect was smaller if the cue was valid than if the cue was invalid. If a cue presented during target motion or after the target vanished indicated the location where that target had appeared, the onset repulsion effect was eliminated. The data (1) suggest that positional uncertainty might contribute to the onset repulsion effect, (2) provide the first evidence of an effect of expectancy regarding target trajectory on the onset repulsion effect, and (3) are partially consistent with previous data involving effects of attention and spatial cuing on the Fröhlich effect and on representational momentum.
\end{abstract}

Keywords Onset repulsion effect · Displacement - Spatial cuing · Attention - Spatial cognition · Spatial memory . Spatial localization

Memory for the initial location of a moving target can be displaced in the direction opposite to subsequent target motion (i.e., a target is judged to have appeared at a location that is shifted away from the actual initial location of that target and in

T. L. Hubbard $(\bowtie)$

Department of Psychology, Texas Christian University,

Fort Worth, TX 76129, USA

e-mail: timothyleehubbard@gmail.com

S. E. Ruppel

Department of Psychology,

University of South Carolina - Upstate,

Spartanburg, SC 29303, USA the direction opposite to subsequent target motion; e.g., ActisGrosso \& Stucchi, 2003), and this backward displacement has been referred to as the onset repulsion effect (Thornton, 2002). Alternatively, memory for the initial location of a moving target can be displaced in the direction of subsequent target motion, and this forward displacement has been referred to as the Fröhlich effect (for a review, see Kerzel, 2010). An onset repulsion effect is more likely to occur if the target is on a blank background (Hubbard \& Motes, 2002), is further away from a boundary (Hubbard \& Motes, 2005), or moves at a relatively slower velocity (Kerzel \& Gegenfurtner, 2004), or if the observer's response involves pointing (Kerzel, 2002). Although the onset repulsion effect has been contrasted with the Fröhlich effect (e.g., Hubbard \& Motes, 2005; Kerzel, 2002; Müsseler \& Kerzel, 2004), variables influencing the onset repulsion effect have received less investigation than have variables influencing the Fröhlich effect or other types of spatial mislocalization. One variable that influences spatial mislocalization is attention, and the experiments reported here examined how changes in the allocation of attention (by spatial cuing) to the initial location of the target influence the onset repulsion effect.

Thornton (2002) suggested several possible mechanisms for the onset repulsion effect. One possible mechanism involves an overcompensation for uncertainty regarding the initial (onset) position of the target, and Thornton suggested that manipulating positional uncertainty might be one way to evaluate this potential mechanism. In a test of this, Müsseler and Kerzel (2004) presented a moving target that appeared at (1) one of two peripheral locations that were $7^{\circ}$ to the left side or right side of a central fixation point or (2) a random location anywhere within a display $14^{\circ}$ in width. The remembered initial location of the target was displaced in the direction opposite to target motion (an onset repulsion effect) in the latter condition but was displaced 
in the direction of target motion (a Fröhlich effect) in the former condition. Müsseler and Kerzel suggested that increased positional uncertainty regarding the initial location of the target in the latter condition resulted in an onset repulsion effect (but see Müsseler, Stork, \& Kerzel, 2008). Hubbard and Motes (2005) presented a moving target inside a larger enclosing stationary frame, and they found a Fröhlich effect if the target initially appeared near a side of the frame (as that side could function as a landmark and positional uncertainty regarding the initial location was decreased) but an onset repulsion effect if the target initially appeared further from a side of the frame (and positional uncertainty regarding the initial location was increased).

A more direct test of the positional uncertainty hypothesis could compare the onset repulsion effect when a spatial cue that indicated where the target would appear was presented (thus eliminating positional uncertainty regarding the initial location of the target) with the onset repulsion effect when no such cue was presented. Although the effect of such a spatial cue on the onset repulsion effect has not been reported, the effect of such a spatial cue on the Fröhlich effect has been reported. Müsseler and Aschersleben (1998) presented a cue in the form of parallel lines above and below the location where a horizontally moving target would appear. The target appeared on the left side or right side of the display; in a valid cue condition, the cue indicated where the target actually appeared, and in an invalid cue condition, the cue indicated a location on the side of the display opposite to where the target actually appeared. The Fröhlich effect was decreased when valid cues were presented, and this was suggested to reflect differences in the time required to shift attention from the cue to the target. A valid cue was closer to the target, and so less time was required for such a shift of attention; as a result, a target preceded by a valid cue entered perceptual awareness more quickly. Similarly, Kirschfeld and Kammer (1999) suggested that focusing visual attention on the location where a target would appear shortened the latency to perceptual awareness of that target, thus allowing the target to be perceived earlier in its trajectory and resulting in a smaller Fröhlich effect.

It is not clear what a theory that is based on latency to perceptual awareness in the Fröhlich effect would predict regarding the effect of a spatial cue on the onset repulsion effect, nor is it clear how or why decreasing latency to perceptual awareness to allow the target to be perceived earlier in its trajectory would result in a displacement in the direction opposite to that trajectory. Given that displacement in the onset repulsion effect extends the length of the represented trajectory, perhaps consideration of another type of displacement in which the length of the represented trajectory is also extended might be more useful. Memory for the final location of a moving target is displaced forward from the actual final location of that target (thus extending the length of the represented trajectory), and this is referred to as representational momentum (for a review, see Hubbard, 2005). In representational momentum, as in onset repulsion, the target is remembered as occupying a location that the target never actually occupied. Hubbard, Kumar, and Carp (2009) reported that if a cue was presented during or after target motion and indicated where the target would vanish or had vanished, displacement in memory for the final location of that target was decreased. Just as an increase in attention to the final location of a target decreased displacement (representational momentum) for the final location of that target, perhaps an increase in attention to the initial location of a target might decrease displacement (onset repulsion) for the initial location of that target.

Closer examination of the literature on representational momentum suggests contrasting predictions regarding the effects of a spatial cue on memory for the initial location of a target. ${ }^{1}$ Hayes and Freyd (2002) presented nontarget stimuli during target presentation and found that forward displacement of the target increased, relative to when nontarget stimuli were not presented. They suggested that displacement is an automatic process and that attention is necessary to stop displacement. This suggestion is consistent with Hubbard et al.'s (2009) findings that cuing the final location of the target led to a decrease in representational momentum and leads to a prediction that increasing the attention allocated to the initial location of the target by spatially cuing that location should decrease onset repulsion. However, Kerzel (2003) presented distractor stimuli during the retention interval between when the target vanished and when the probe appeared and found that forward displacement of the target was eliminated or decreased, relative to when distractor stimuli were not presented. He suggested that displacement is not an automatic process

\footnotetext{
${ }^{1}$ Although the present experiments focus on spatial cues, other types of cues might be possible. For example, Teramoto, Hidaka, Gyoba, and Suzuki (2010) presented an auditory tone concurrent with motion of a visual target, and they referred to this tone as a "temporal cue." The tone ended slightly before the visual target vanished, when the visual target vanished, or slightly after the visual target vanished. Representational momentum of the visual target decreased if the tone ended before the visual target vanished and increased if the tone ended after the target vanished, but only if the onset of the tone was synchronized with onset of the visual target. Although cuing the time when a target would vanish could, in principle, allow a participant to determine the location at which the target would vanish, it is not clear whether the auditory tone in Teramoto et al. (1) was intended or used as a cue regarding disappearance of the visual target or (2) influenced allocation of attention to the location of the target in the same way that a spatial cue would. Also, given that tones in Teramoto et al. influenced visual representational momentum only if the onset of the tones was synchronized with the onset of the visual target, it is possible that the synchronized tones were considered as part of the (multimodal) target, rather than as a separate and distinct cue, whereas the spatial cues in the present experiments were clearly separate and distinct from the targets.
} 
and that attention is necessary in order to generate and maintain displacement. This suggestion leads to a prediction that increasing the attention allocated to the initial location of the target by spatially cuing that location should maintain (if there is a threshold of attention above which a constant level of displacement occurs) or increase (if attention and displacement are monotonically related) onset repulsion.

In the experiments reported here, a cue indicating the initial location of a target was presented before the target appeared, during target motion, or after the target vanished. Presenting a spatial cue should increase the allocation of attention to the location where the target would appear or had appeared (cf. Tsal \& Bareket, 1999, 2005), and such an increase in attention to the initial target location should decrease positional uncertainty. If the positional uncertainty hypothesis is correct, or if displacement involves an automatic process that can be stopped by an allocation of attention, the onset repulsion effect should be decreased if the initial location of the target is cued. Alternatively, if displacement is generated or maintained by an allocation of attention, the onset repulsion effect should stay the same or be increased if the initial location of the target is cued. In Experiment 1, a cue at the initial target location was presented before the target appeared on some trials, and a cue was not presented on other trials. In Experiment 2, a cue was presented before the target appeared, and the cue was either at the location where the target would appear (a valid cue) or at a location on the opposite side of the display from where the target would appear (an invalid cue). In Experiment 3, a cue at the initial target location was presented during target motion on some trials, and a cue was not presented on other trials. In Experiment 4, a cue at the initial target location was presented during the retention interval after the target vanished and before a probe appeared on some trials, and a cue was not presented on other trials.

\section{Experiment 1}

Participants viewed rightward or leftward implied motion of a target on a featureless background. On half of the trials, a cue was briefly presented before the target appeared. The cue indicated the location at which the target subsequently appeared, and the cue vanished before the target appeared. On half of the trials, a cue was not presented. The spatial location where the target would appear should be allocated more attention (and positional uncertainty correspondingly reduced) if a cue was presented than if a cue was not presented. After the target vanished, a stationary probe was presented, and the location of the probe was either (1) shifted backward from the initial location of the target, (2) the same as the initial location of the target, or (3) shifted forward from the initial location of the target. Participants judged whether the probe was at the same location where the target appeared or at a different location, and calculation of a weighted mean based on the probabilities of same responses to different probe positions allowed estimation of any potential displacement in the remembered initial location of the target. The weighted mean estimates of displacement and the probabilities of same responses to different probe locations if a cue was presented were compared with the weighted mean estimates of displacement and the probabilities of same responses to different probe locations if a cue was not presented.

\section{Method}

Participants The participants were 15 undergraduates who participated for partial course credit and were naive as to the hypotheses.

Apparatus The stimuli were displayed upon and the data were collected with a Gateway desktop computer connected to a 15 -in. color monitor with a refresh rate of $60 \mathrm{~Hz}$ and a resolution of $1,024 \times 768$ pixels. Participants' head and eye movements were not constrained, and the average viewing distance was approximately $60 \mathrm{~cm}$.

Stimuli The moving target and probe were black squares 20 pixels (approximately $0.83^{\circ}$ of visual angle) in width and in height and were presented on a white background. As is shown in Fig. 1, on each trial, there were five successive presentations of the target that implied consistent rightward motion of the target or consistent leftward motion of the target, ${ }^{2}$ and these successive presentations are referred to as inducing stimuli. Each inducing stimulus was presented for $250 \mathrm{~ms}$, and there was a $250-\mathrm{ms}$ interstimulus interval (ISI) between successive inducing stimuli. For rightward motion, the first inducing stimulus appeared approximately midway between the left side and the center of the display, and the horizontal coordinates of each successive inducing stimulus were located 40 pixels (approximately $1.66^{\circ}$ of visual angle) to the right of the previous inducing stimulus; for leftward motion, the first inducing stimulus appeared approximately midway between the right side and the

\footnotetext{
$\overline{2}$ Thornton (2002) found an onset repulsion effect with smooth motion and with implied motion, and the magnitude of the onset repulsion effect was larger with smooth motion. Although Thornton rejected the possibility that an eye movement mechanism was responsible for the onset repulsion effect, it has been shown that eye movements can modulate representational momentum with smooth motion (Kerzel, 2000), but not with implied motion (Kerzel, 2003). In order to avoid the possibility that an eye movement mechanism might modulate displacement in the present experiments, implied motion was used. Use of implied motion also makes targets in the present experiments more comparable with targets in Hubbard et al.'s (2009) study of the effects of spatial cuing on representational momentum.
} 


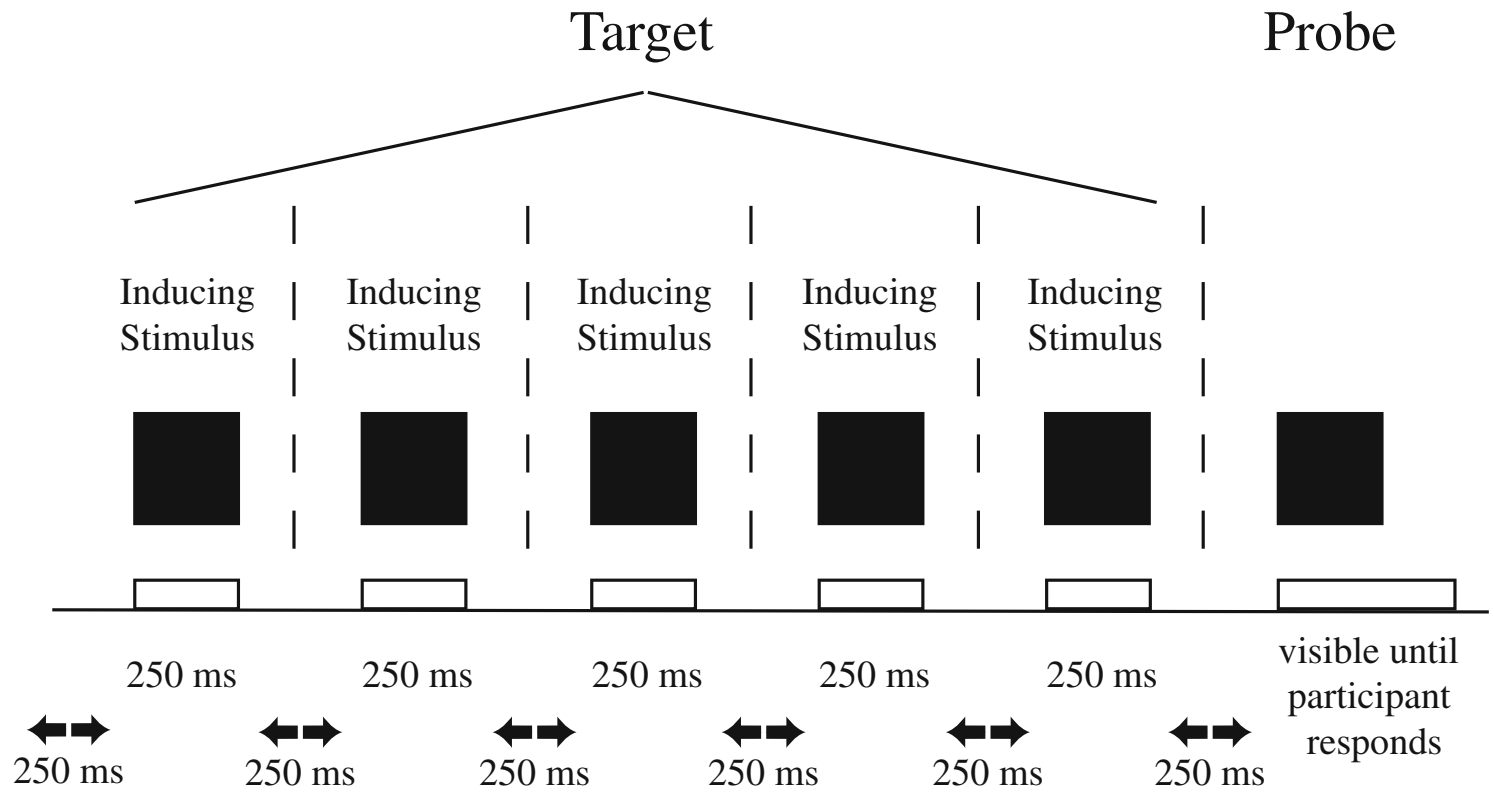

Cue

Exps. 1 and 2

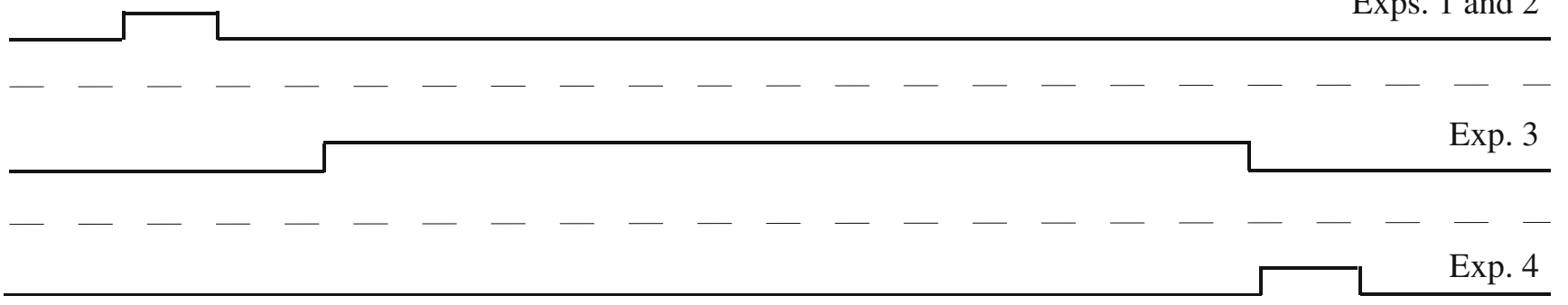

Fig. 1 Structure of the experimental trials in Experiments 1, 2, 3, and 4. The top of the figure illustrates the target and probe stimuli. There were five inducing stimuli that comprised the target; each inducing stimulus was presented for $250 \mathrm{~ms}$, and there was a $250-\mathrm{ms}$ interstimulus interval between successive inducing stimuli. The probe was presented after a retention interval of $250 \mathrm{~ms}$ and remained visible until the participant

center of the display, and the horizontal coordinates of each successive inducing stimulus were located 40 pixels to the left of the previous inducing stimulus. The vertical coordinates of the inducing stimuli were approximately centered along the vertical axis of the display.

The probe was presented at the same vertical coordinates as the moving target, and the probe was located at one of nine horizontal positions relative to the initial location of the moving target (i.e., the location of the first inducing stimulus): $-16,-12,-8,-4,0,+4,+8,+12$, or +16 pixels. Probe positions denoted by a minus sign indicated that the probe was backward (i.e., shifted in the direction opposite to target motion) from the initial location of the target by responded. The bottom of the figure illustrates the cue stimulus and the timing of cue presentation, relative to the target and probe presentation in each of the experiments, and the raised portions of the horizontal lines indicate when the cue was presented in each experiment (i.e., before the target appeared in Experiments 1 and 2, during target motion in Experiment 3, after the target vanished in Experiment 4)

the indicated number of pixels, and probe positions denoted by a plus sign indicated that the probe was forward (i.e., shifted in the direction of target motion) from the initial location of the target by the indicated number of pixels; the zero probe position was the same as the initial location of the target. The cue was a red plus sign, and each horizontal or vertical arm of the plus sign was 10 pixels long and 4 pixels thick (the total width and height of the cue was 20 pixels, the same as the width and height of the inducing stimuli and probe). Each participant received 216 trials (2 cues [present, absent] $\times 9$ probes $[-16,-12,-8,-4$, $0,+4,+8,+12,+16] \times 2$ directions [leftward, rightward] $\times 6$ replications) in a different random order. 
Procedure Participants were first given a practice session consisting of 10 practice trials that were randomly drawn from the experimental trials. On trials on which a cue was presented, there was a 1,000-ms pause, the cue appeared for $250 \mathrm{~ms}$, the display cleared, and there was a pause of $250 \mathrm{~ms}$ before the first inducing stimulus appeared. On trials on which a cue was not presented, there was a 1,500-ms pause before the first inducing stimulus appeared. After the final inducing stimulus vanished, there was a retention interval of $250 \mathrm{~ms}$ before the probe appeared. After the probe appeared, participants pressed a key marked $S$ or a key marked $D$ (the $M$ and $C$ keys, respectively, of a standard keyboard) to indicate whether the location of the probe was the same as or different from the initial location of the moving target. Participants then initiated the next trial.

\section{Results}

Probabilities of a same response for each probe position are shown in Fig. 2. Two types of analyses were performed: an examination of the weighted mean estimates of displacement and an examination of the probabilities of same responses. A weighted mean significantly smaller than zero for a given condition would indicate that an onset repulsion effect occurred for that condition, and a weighted mean significantly larger than zero for a given condition would indicate that a Fröhlich effect occurred for that condition. The heights and the shapes of the distributions of the probabilities of same responses as a function of probe position indicated the relative uncertainty of participants and, in conjunction with the weighted means, allowed evaluation of the positional uncertainty hypothesis.

Weighted means Consistent with previous studies of displacement in memory for the spatial location of a target (e.g.,

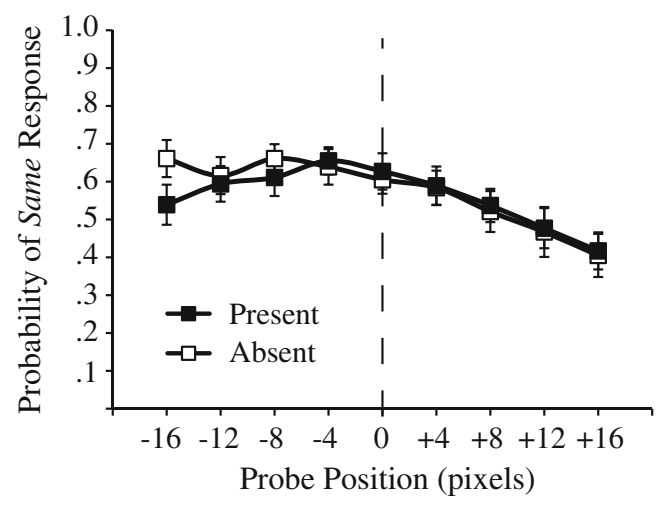

Fig. 2 The probability of a same response as a function of probe position in Experiment 1. Responses when the cue was present are shown by filled (black) squares, and responses when the cue was absent are shown by the open (white) squares. Error bars indicate standard errors of the means
Hayes \& Freyd, 2002; Hubbard et al., 2009; Munger, Solberg, Horrocks, \& Preston, 1999), estimates of the direction and magnitude of displacement were determined by calculating a weighted mean (the sum of the products of the proportion of same responses and the distance of the probe from the initial location of the target, in pixels, divided by the sum of the proportions of same responses) for each participant for each condition. The sign of a weighted mean indicated the direction of displacement (i.e., a minus sign indicated displacement in the direction opposite to target motion; a plus sign indicated displacement in the direction of target motion), and the absolute value of a weighted mean indicated the magnitude of displacement (i.e., a larger absolute value indicated a larger magnitude of displacement).

Weighted means if the cue was present $(M=-1.04)$, $t(14)=-2.24, p<.021$, and if the cue was absent $(M=$ $-1.80), t(14)=-3.23, p<.003$, were significantly less than zero. Weighted means were also analyzed in a 2 (cue) $\times 2$ (direction) repeated measures ANOVA. Cue was significant, $F(1,14)=7.06, M S E=1.24, p<.001$, such that presence of the cue resulted in a smaller magnitude of negative displacement than did absence of the cue. Neither direction nor cue $\times$ direction was significant.

Probabilities of same responses Same/different judgments were analyzed in a $2($ cue $) \times 2$ (direction) $\times 9$ (probe) repeated measures ANOVA. Cue did not influence probability of a same response, $F(1,14)=0.45, M S E=0.05, p>.51$, nor did cue significantly interact with any other factors, $F_{\mathrm{S}}<1.31$, $p \mathrm{~s}>.27$. Probe was significant, $F(8,112)=5.14, M S E=$ $0.08, p<.0001$, and interacted with direction, $F(8,112)=$ 2.54, MSE $=0.05, p<.02$; there were slightly higher probabilities of same responses for negative probe positions than for positive probe positions (see Fig. 2), and the differences between probabilities of same responses for negative probes and probabilities of same responses for positive probes were slightly larger for leftward motion than for rightward motion. No other main effects or interactions were significant.

\section{Discussion}

An onset repulsion effect occurred regardless of whether the cue was present or absent, and the onset repulsion effect was smaller if the cue was present than if the cue was absent. An increase in the allocation of attention (directed by the cue) to the location where the target would appear decreased the onset repulsion effect, and this is (1) consistent with the hypothesis that onset repulsion is an automatic process and that an increase in the allocation of attention can stop such displacement and (2) not consistent with the hypotheses that attention is necessary to generate 
or maintain onset repulsion or that onset repulsion results from modular or cognitively impenetrable processes. To the extent that spatial information in the cue provided information regarding the initial portion of the target trajectory, Experiment 1 also demonstrated an effect of expectation regarding target trajectory on the onset repulsion effect; although effects of expectation regarding target trajectory have been previously demonstrated for representational momentum (Hubbard \& Bharucha, 1988; Johnston \& Jones, 2006; Verfaillie \& d'Ydewalle, 1991), such an effect has not been previously demonstrated for onset repulsion. Also, participants were more likely to respond same to probes closer to the actual initial location of the target, but the presence of the cue did not influence the overall probability of a same response or interact with other variables. Such a dissociation between the probability of a same response and the magnitude of onset repulsion does not appear consistent with the positional uncertainty hypothesis.

An examination of Fig. 2 reveals that probabilities of same responses for the cue-absent condition and for the cue-present condition were still relatively high even for the most distant probes, and this suggests that the range of probe distances might not have been optimal. As a consequence, the range of probabilities across probe distances is narrow, and this would have presumably made it more difficult to obtain the asymmetry in the probabilities of same responses between negative probes and positive probes that would indicate a consistent direction of displacement; even so, a significant onset repulsion effect was found, and this suggests that onset repulsion is relatively robust. Along these lines, although a narrow range of probe distances could obscure an effect that is present, a narrow range of probe distances is unlikely to indicate an effect that is not present. Although the addition of probes more distant from the actual initial location of the target would presumably have resulted in a stronger statistical effect, the range of probe distances used in Experiment 1 was sufficient to reveal a significant onset repulsion effect and to reveal the influence of the cue on onset repulsion. Also, findings that a spatial cue that indicated the initial location of the target decreased but did not eliminate displacement in memory for the initial location of the target paralleled previous findings involving the effect of a spatial cue on the Fröhlich effect (Müsseler \& Aschersleben, 1998) and on representational momentum (Hubbard et al., 2009).

\section{Experiment 2}

In Experiment 1, the cue was presented at the same location where the target subsequently appeared, and the presence of the cue decreased the onset repulsion effect. This decrease presumably resulted from an increase in the allocation of attention to the initial location of the target that was based on spatial information provided by the cue. If effects of the cue in Experiment 1 were due to spatial information regarding where the target would appear, it could be predicted that the onset repulsion effect if the cue was valid (i.e., if the cue was presented in the location where the target subsequently appeared) should be less than the onset repulsion effect if the cue was invalid (i.e., if the cue was presented in a location different from where the target subsequently appeared). Alternatively, if the cue merely provided an alerting stimulus to participants regarding the impending appearance of the target and spatial information in the cue was not used by participants, it could be predicted that the onset repulsion effect should not be influenced by whether spatial information in the cue was valid or invalid. Accordingly, in Experiment 2, a cue was presented before the target appeared on each trial. On $75 \%$ of the trials, the cue was valid and was presented at the location where the target subsequently appeared, and on $25 \%$ of the trials, the cue was invalid and was presented on the opposite side of the display from where the target subsequently appeared.

\section{Method}

Participants The participants were 14 undergraduates from the same participant pool as in Experiment 1, and none had participated in the previous experiment.

Apparatus The apparatus was the same as in Experiment 1.

Stimuli The moving targets and probes were the same as in Experiment 1. The cue was the same as in Experiment 1, with the following exceptions: On $75 \%$ of the trials, the cue was presented at the location where the target on that trial would appear, and on $25 \%$ of the trials, the cue was presented on the opposite side of the display (in a plausible location corresponding to where targets on other trials had appeared or would appear). Each participant received 288 trials $(2$ cues [valid, invalid] $\times 9$ probes [-16, $-12,-8,-4,0,+4,+8,+12,+16] \times 2$ directions [leftward, rightward] $\times 8$ replications [6 of valid trials, 2 of invalid trials]) in a different random order.

Procedure The procedure was the same as that for trials on which the cue appeared in Experiment 1.

\section{Results}

Probabilities of a same response for each probe position are shown in Fig. 3, and analyses of weighted means and of probabilities of same responses were performed. 
Weighted means Weighted means based on the probabilities of same responses were calculated as in Experiment 1. Weighted means if the cue was valid $(M=-1.31), t(13)=$ $-2.71, p<.02$, and if the cue was invalid $(M=-2.30), t(13)=$ $-4.17, p<.002$, were significantly less than zero. Weighted means were also analyzed in a 2 (cue) $\times 2$ (direction) repeated measures ANOVA. Cue was significant, $F(1,13)=6.01$, $M S E=2.30, p<.03$, and valid cues resulted in a smaller magnitude of negative displacement than did invalid cues. Neither direction nor cue $\times$ direction was significant.

Probabilities of same responses Same/different judgments were analyzed in a 2 (cue) $\times 2$ (direction) $\times 9$ (probe) repeated measures ANOVA. Cue influenced the probability of a same response, $F(1,13)=7.51, M S E=0.37, p<.02$, such that the probability of a same response was higher for valid cues $(M=.67)$ than for invalid cues $(M=.51)$. Probe was significant, $F(8,104)=5.75, M S E=0.08, p<.0001$, with slightly higher probabilities of same responses to negative probe positions than to positive probe positions. No other main effects or interactions were significant.

\section{Discussion}

An onset repulsion effect occurred regardless of whether the cue was valid or invalid, and the onset repulsion effect was smaller if the cue was valid than if the cue was invalid. Differences in the onset repulsion effect as a function of cue validity suggest that the effect of cue presentation in Experiment 1 was due, at least in part, to the specific spatial information provided by the cue and was not due solely to the cue providing a general alerting signal that the target was about to appear. The smaller displacement if a valid cue was presented is consistent with the hypothesis that the effect

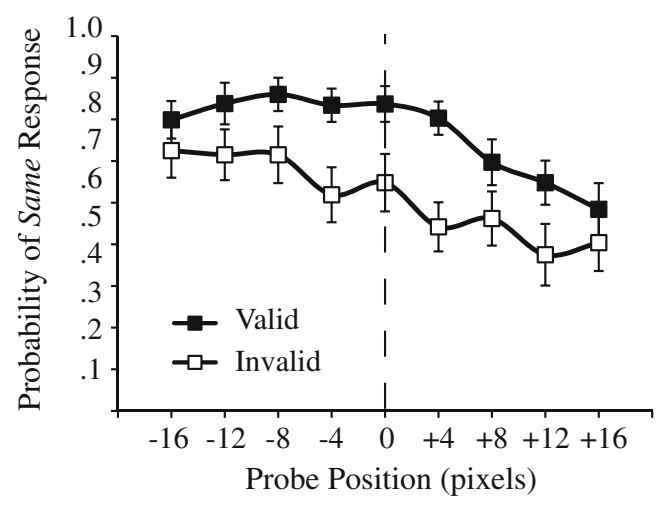

Fig. 3 The probability of a same response as a function of probe position in Experiment 2. Responses when the cue was valid are shown by filled (black) squares, and responses when the cue was invalid are shown by the open (white) squares. Error bars indicate standard errors of the means of the cue in Experiment 1 and of the valid cue in Experiment 2 was due to an increased allocation of attention to the spatial location indicated by the cue. Consistent with the existence of the onset repulsion effect and with the negative weighted means, the probabilities of same responses appear higher for negative probes than for positive probes. Consideration of the positional uncertainty hypothesis predicts higher probabilities of same responses with invalid cues than with valid cues (as spatial uncertainty regarding the initial location of the target would be higher following an invalid cue, participants would be less able to discriminate between probes at or near the actual initial location, and so the overall probability of a same response would increase); however, the probability of a same response was actually higher with valid cues than with invalid cues. Thus, the results of Experiment 2 do not appear consistent with the positional uncertainty hypothesis.

The onset repulsion effect if a cue was presented in Experiment 1 and if a valid cue was presented in Experiment 2 appears smaller than the onset repulsion effect if a cue was not presented in Experiment 1, and the onset repulsion effect if a cue was not presented in Experiment 1 appears smaller than the onset repulsion effect if an invalid cue was presented in Experiment 2. This pattern is consistent with previous findings in the attention literature that (1) a valid cue can facilitate the processing of a subsequently presented target that appears at the cued location and (2) an invalid cue can interfere with the processing of a subsequently presented target that appears at a noncued location (e.g., Posner, Nissen, \& Ogden, 1977). Additionally, the decrease in the onset repulsion effect with valid cues relative to invalid cues in Experiment 2 is consistent with (1) the decreased Fröhlich effect with valid cues relative to invalid cues in Müsseler and Aschersleben (1998) and (2) the hypothesis that a spatial cue can act as a prime to facilitate processing of the target if the target subsequently occupies the location that has been cued (cf. effects of high-relevant cues and low-relevant cues on representational momentum in Hubbard et al., 2009). Also, there was a larger separation between the functions for valid cues and for invalid cues shown in Fig. 3 than between the functions for cue absent and for cue present shown in Fig. 2, and this is consistent with "cue present" being analogous to "valid" and "cue absent" being more analogous to "neutral" than to "invalid."

\section{Experiment 3}

In Experiments 1 and 2, a cue presented before the target appeared, and at the location where the target would subsequently appear, led to a reduction, but not to an elimination, of the onset repulsion effect. If the strength or 
amount of attention allocated to the cued location decays or otherwise decreases with the passage of time, effects of an initial allocation of attention to the cue might be diminished by the relatively long latency between when the cue was presented and when participants responded. Presentation of the cue during target motion would decrease the latency between when the cue was presented and when participants responded, and so if the allocation of attention decayed or otherwise decreased with longer latencies between when the cue was presented and when participants responded, presentation of the cue during target motion could be predicted to further decrease or even eliminate the onset repulsion effect. Alternatively, presentation of a cue during target motion could interfere with the ability to limit displacement of the initial location of the target (cf. Hayes \& Freyd, 2002, for an analogous suggestion involving presentation of a nontarget stimulus during target motion on displacement of the final location of the target), and so presentation of a cue during target motion could be predicted to increase the onset repulsion effect. Accordingly, on half of the trials in Experiment 3, a cue indicating the initial location of the target was presented during target motion, and on half of the trials, no cue was presented.

\section{Method}

Participants The participants were 14 undergraduates from the same participant pool as in Experiment 1, and none had participated in the previous experiments.

Apparatus The apparatus was the same as in Experiment 1.

Stimuli The moving targets and probes were the same as in Experiment 1. The cue was the same as in Experiment 1, with the following exception: On half of the trials, the cue was presented during target motion, and on half of the trials, no cue was presented. A cue that appeared or vanished during target presentation might evoke apparent motion between that cue and an inducing stimulus or might otherwise distort or interfere with the perception of target motion, and so if a cue was presented, that cue appeared when the first inducing stimulus appeared and vanished when the final inducing stimulus vanished. ${ }^{3}$ Each participant received 216 trials $(2$ cues [present, absent] $\times 9$ probes

\footnotetext{
${ }^{3}$ Had the cue been presented immediately after target onset and for a duration equal to the cues in Experiments 1 and 2, it is not clear whether the cue would have contributed any additional information beyond that available from perception of the first inducing stimulus that was simultaneously visible. Had the cue been presented for a duration equal to the cues in Experiments 1 and 2 and by a temporal separation from target onset equal to the cues in Experiments 1 and 2, the cue would have appeared during the ISI between the first and second inducing stimuli, and the risk of apparent motion between the cue and an inducing stimulus would have been much greater.
}

$[-16,-12,-8,-4,0,+4,+8,+12,+16] \times 2$ directions [leftward, rightward] $\times 6$ replications) in a different random order.

Procedure The procedure was the same as in Experiment 1, with the following exceptions: The first inducing stimulus appeared 1,000 ms after participants pressed the key to begin the trial. If a cue was presented on a trial, the cue appeared when the first inducing stimulus appeared (and the cue was superimposed on the first inducing stimulus and was clearly visible), was continually visible during presentation of the inducing stimuli, and vanished when the final inducing stimulus vanished.

\section{Results}

Probabilities of a same response for each probe position are shown in Fig. 4, and analyses of weighted means and of probabilities of same responses were performed.

Weighted means Weighted means based on the probabilities of same responses were calculated as in Experiment 1. Weighted means if the cue was present $(M=-0.71), t(13)=-1.13, p>.14$, did not differ from zero, and weighted means if the cue was absent $(M=-1.27), t(13)=-1.96, p<.04$, were significantly less than zero. Weighted means were also analyzed in a 2 (cue) $\times 2$ (direction) repeated measures ANOVA. Cue was not significant, $F(1,13)=0.75, M S E=5.95, p>.40$, although the means were consistent with a weak trend for the presence of the cue to result in a smaller magnitude of negative displacement, and this would be consistent with the effect of cue in Experiment 1. Neither direction nor cue $\times$ direction was significant.

Probabilities of same responses Same/different judgments were analyzed in a 2 (cue) $\times 2$ (direction) $\times 9$ (probe)

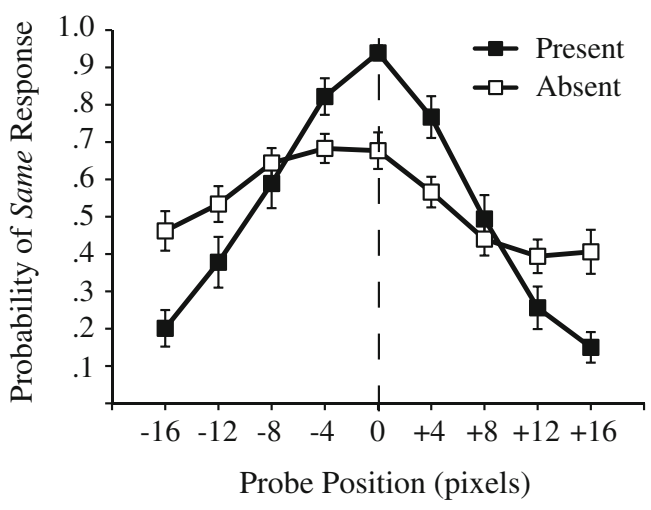

Fig. 4 The probability of a same response as a function of probe position in Experiment 3. Responses when the cue was present are shown by filled (black) squares, and responses when the cue was absent are shown by the open (white) squares. Error bars indicate standard errors of the means 
repeated measures ANOVA. Cue did not influence probability of a same response, $F(1,14)=0.18, M S E=0.42, p>$ .68. Probe was significant, $F(8,112)=28.03, M S E=0.82$, $p<.0001$, and probe interacted with cue, $F(8,112)=9.89$, $M S E=0.06, p<.0001$, and with direction, $F(8,112)=$ 4.52, MSE $=0.04, p<.001$, such that decreases in the probability of a same response with increases in probe distance were larger if a cue was present (see Fig. 4). No other main effects or interactions were significant.

\section{Discussion}

An onset repulsion effect occurred if a cue was not presented, and this is consistent with Experiment 1. However, if a cue that indicated the initial location of the target appeared when the target appeared and vanished when the target vanished, an onset repulsion effect did not occur. The longer cue presentation (during target motion) in Experiment 3 might have enabled a better encoding of the initial location of the target than did the briefer cue presentation (before the target appeared) in Experiment 1. The elimination of the onset repulsion effect if a cue was presented during target motion in Experiment 3, coupled with a reduction but not elimination of the onset repulsion effect if a cue was presented before the target appeared in Experiment 1, is consistent with the hypotheses that (1) decreasing the latency between when a cue is presented and when the participant responds increases the effect of that cue and (2) attention maintains or generates displacement, because a cue presented during target motion will presumably result in a decrease in the allocation of attention to the target, relative to the allocation of attention to the target in the absence of a cue during target motion. Alternatively, elimination of the onset repulsion effect if a cue was presented during target motion might reflect participants' ignoring the target and attending only to the cue, and this possibility will be further addressed in the discussion of Experiment 4.

Whether the cue was presented during target motion did not influence the overall probability of a same response, and so it might appear that the elimination of the onset repulsion effect if a cue was presented in Experiment 3 was not related to positional uncertainty regarding the initial location of the target. However, whether the cue was presented during target motion interacted with probe position such that probabilities of same responses increased for closer probes and decreased for distant probes if the cue was presented (see Fig. 4), and this interaction suggests that cue presentation resulted in a decrease in positional uncertainty regarding the initial location of the target. The interaction of cue presentation with probe position in Experiment 3, coupled with the lack of a similar interaction of cue presentation with probe position in Experiment 1, suggests the cue was more effective in decreasing positional uncertainty regarding the initial location of the target in Experiment 3 than in Experiment 1 (cf. differences between the cue-present and cue-absent distributions in Figs. 2 and 4). The elimination of the onset repulsion effect in Experiment 3 and the reduction of the onset repulsion effect in Experiment 1, in conjunction with the interaction of cue presentation and probe position in Experiment 3 and the absence of a similar interaction in Experiment 1, is consistent with the positional uncertainty hypothesis; a larger decrease in positional uncertainty was associated with a larger decrease of the onset repulsion effect.

\section{Experiment 4}

In Experiments 1 and 2, presentation of a cue that indicated the initial location of the target decreased but did not eliminate the onset repulsion effect in memory for the initial location of that target. In Experiment 3, presentation of a cue that indicated the initial location of the target eliminated the onset repulsion effect in memory for the initial location of the target. The larger effect of cue presentation on the onset repulsion effect in Experiment 3 than in Experiments 1 and 2 might reflect the closer temporal proximity of cue presentation to participants' responding in Experiment 3 than in Experiments 1 and 2. Alternatively, the cue in Experiment 3 was presented for a longer duration than were the cues in Experiments 1 and 2. If the greater duration of the cue in Experiment 3 resulted in elimination of the onset repulsion effect, cues with the same duration as cues in Experiments 1 and 2 should not eliminate the onset repulsion effect if those cues are presented closer in time to when participants respond. However, if the closer temporal proximity to participants' responding resulted in elimination of the onset repulsion effect in Experiment 3, cues with the same duration as cues in Experiments 1 and 2 should eliminate the onset repulsion effect if those cues are presented closer in time to when participants respond. Accordingly, on half of the trials in Experiment 4, a cue with the same duration as the cues in Experiments 1 and 2 was presented during the retention interval, and on half of the trials, no cue was presented.

Method

Participants The participants were 15 undergraduates from the same participant pool as in Experiment 1, and none had participated in the previous experiments.

Apparatus The apparatus was the same as in Experiment 1. 
Stimuli The moving targets and probes were the same as in Experiment 1. The cue was the same as in Experiment 1, with the following exceptions: On half of the trials, the cue was presented during the retention interval between when the final inducing stimulus vanished and when the probe appeared, and on half the trials, no cue was presented. Each participant received 216 trials $(2$ cues [present, absent] $\times 9$ probes $[-16,-12,-8,-4,0,+4,+8,+12,+16] \times 2$ directions [leftward, rightward] $\times 6$ replications) in a different random order.

Procedure The procedure was the same as in Experiment 3, with the following exceptions: If a cue was presented on a trial, the cue appeared when the final inducing stimulus vanished and was visible for $250 \mathrm{~ms}$ (the same as the duration of the retention interval and the duration of cue presentation in Experiments 1 and 2), and then the cue vanished when the probe appeared.

\section{Results}

Probabilities of a same response for each probe position are shown in Fig. 5, and analyses of weighted means and of probabilities of same responses were performed.

Weighted means Weighted means based on the probabilities of same responses were calculated as in Experiment 1. Weighted means if the cue was present $(M=-0.75)$, $t(14)=-1.65, p<.13$, did not differ from zero, and weighted means if the cue was absent $(M=-0.96), t(14)=$ $-2.95, p<.02$, were significantly less than zero. Weighted means were also analyzed in a 2 (cue) $\times 2$ (direction) repeated measures ANOVA. Cue was not significant, $F(1,14)=0.20$, $M S E=3.61, p>.66$, although the means were consistent with a weak trend for the presence of the cue to result in a

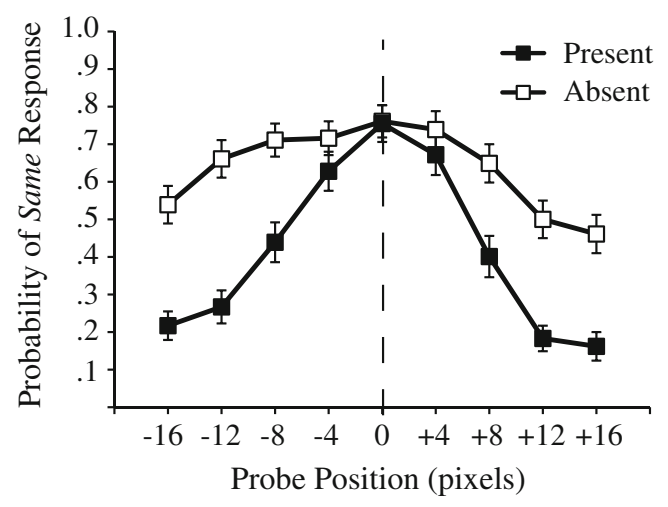

Fig. 5 The probability of a same response as a function of probe position in Experiment 4. Responses when the cue was present are shown by filled (black) squares, and responses when the cue was absent are shown by the open (white) squares. Error bars indicate standard errors of the means smaller magnitude of negative displacement, and this would be consistent with the effect of cue in Experiment 1 . Direction, $F(1,14)=3.95, M S E=4.55, p<.10$, was marginally significant, with a trend for a larger displacement for leftward motion than for rightward motion if the cue was present. Cue $\times$ direction was not significant.

Probabilities of same responses Same/different judgments were analyzed in a 2 (cue) $\times 2$ (direction) $\times 9$ (probe) repeated measures ANOVA. Cue influenced probability of a same response, $F(1,14)=14.05, M S E=0.48, p<.003$, such that probability of a same response was lower if a cue was present $(M=.41)$ than if a cue was absent $(M=.64)$. Probe was significant, $F(8,112)=23.69, M S E=0.07$, $p<.0001$, and interacted with cue, $F(8,112)=7.62, M S E=$ $0.04, p<.0001$, and with direction, $F(8,112)=2.04$, $M S E=0.05, p<.05$, such that decreases in the probability of a same response with increases in probe distance were larger if a cue was present (see Fig. 5) and for leftward motion. No other main effects or interactions were significant.

\section{Discussion}

Consistent with Experiments 1 and 3, an onset repulsion effect occurred if a cue was absent, and consistent with Experiment 3, an onset repulsion effect did not occur if a cue was presented. Elimination of the onset repulsion effect if a cue was presented in Experiment 4 is (1) consistent with the hypothesis that elimination of the onset repulsion effect if a cue was presented in Experiment 3 was due to a decrease in the latency between when the cue was presented and when participants responded but (2) not consistent with the hypothesis that elimination of the onset repulsion effect in Experiment 3 if a cue was presented was due to the longer duration of cue presentation in Experiment 3 than in Experiments 1 and 2 (as the cue in Experiment 4 was presented for the same duration as was the cue in Experiments 1 and 2). Thus, the duration of cue presentation is not the critical parameter regarding whether an onset repulsion effect is decreased or eliminated by an increase in allocation of attention to the initial location of the target. Rather, comparison of the findings of Experiments 3 and 4, and comparison of the findings of Experiments 3 and 4 with the findings of Experiments 1 and 2, suggest that the critical parameter regarding whether an onset repulsion effect is decreased or eliminated by a spatial cue that increases allocation of attention to the initial location of the target is whether the cue was presented before or after the target appeared.

Consistent with Experiment 3, the probability of a same response decreased more with increases in probe distance if a cue was presented (see Fig. 5), and as was noted earlier, 
an onset repulsion effect occurred only if a cue was not presented. Additionally, there was a higher overall probability of a same response if a cue was not presented. This configuration of findings is consistent with the positional uncertainty hypothesis. Although it could be suggested that elimination of the onset repulsion effect in Experiment 3 occurred because participants ignored the target if a cue was presented, elimination of the onset repulsion effect in Experiment 4 could not result from participants' ignoring the target if a cue was presented. Participants in Experiment 4 would not know until after the target vanished whether a cue would be presented on that trial, and if participants in Experiment 4 ignored the target and waited for a cue, responses in the cue-absent condition would have been at chance levels. Along these lines, the general similarity of the responses in Experiments 3 and 4 suggests that participants in Experiment 3 did not ignore the target if a cue was presented, and the existence of an onset repulsion effect if a cue was presented in Experiments 1 and 2 suggests that participants in Experiments 1 and 2 did not ignore the target if a cue was presented. In general, the data across experiments are not consistent with a hypothesis that participants ignored the target if a cue was presented.

\section{General discussion}

The onset repulsion effect was (1) decreased but not eliminated if a cue that indicated the location at which the target would appear was presented before the target appeared, (2) decreased if a cue presented before the target appeared provided valid spatial information rather than invalid spatial information regarding the initial location of the target, and (3) eliminated if a cue that indicated the initial location of the target was presented during target motion or during the retention interval between when the target vanished and when the probe appeared. If a cue is considered to increase attention to the cued location, an increase in attention to the initial location of the target (1) decreased the onset repulsion effect if that increase in attention occurred before the target appeared and (2) eliminated the onset repulsion effect if that increase in attention occurred during or after the target appeared. Also, probabilities of a same response (1) were generally higher for probe positions closer to the initial location and for negative probe positions, (2) were generally not influenced by cues that indicated the initial location of the target and that were presented before or during target motion, (3) decreased if a cue that indicated the initial location of the target was presented during the retention interval, (4) decreased more rapidly with increases in probe distance when cues were presented during or after target motion, and (5) increased if a cue presented before the target appeared provided valid spatial information rather than invalid spatial information regarding the initial location of the target.

If increases in the overall probabilities of same responses and smaller decreases in the probabilities of same responses with increases in probe distance reflect increases in positional uncertainty, then the probabilities of same responses, in conjunction with the weighted mean estimates of the magnitude of the onset repulsion effect, can be used to evaluate the hypothesis that the onset repulsion effect is due to increased positional uncertainty regarding the initial location of the target. Although some of the findings in the present experiments are consistent with the positional uncertainty hypothesis (e.g., decreases in the onset repulsion effect if cues were presented in Experiment 1, larger decreases in the probabilities of same responses with increases in probe distance and elimination of the onset repulsion effect if cues were presented in Experiments 3 and 4 , an onset repulsion effect accompanied by a higher probability of a same response in Experiment 4), other findings in the present experiments are not consistent with the positional uncertainty hypothesis (e.g., lack of change in the overall probabilities of same responses with a decrease in or elimination of onset repulsion in Experiments 1 and 3, higher probabilities of same responses with valid cues than with invalid cues in Experiment 2). The magnitude of positional uncertainty appears related to but distinct from the magnitude of the onset repulsion effect, and so although positional uncertainty might contribute to or enable the onset repulsion effect, positional uncertainty does not appear to be the sole underlying mechanism or cause of the onset repulsion effect.

There are at least two hypotheses for why the onset repulsion effect was decreased in Experiments 1 and 2 but eliminated in Experiments 3 and 4. One hypothesis is that the cue interfered (or interfered more) with processing of target information in Experiments 3 and 4 and did not interfere (or interfered less) with processing of target information in Experiments 1 and 2. Presentation of a cue during the retention interval in Experiment 4 might be similar to presentation of a distractor during the retention interval in Kerzel (2003); just as Kerzel found that a distractor presented during the retention interval eliminated representational momentum, Experiment 4 found that a cue presented during the retention interval eliminated onset repulsion. Kerzel hypothesized that the distractor interfered with processing of the target and, thus, disrupted displacement, and it is possible that presentation of the cue during the retention interval in Experiment 4 or during target motion in Experiment 3 similarly interfered with processing of the target and disrupted displacement. The cue was not present during or after target presentation in Experiments 1 and 2 (and participants did not have to maintain a representation of the target during cue presentation), and 
so the cue in Experiments 1 and 2 would not have as strongly interfered with processing of the target. However, interference with processing of the target would presumably increase uncertainty regarding the initial location of the target, and examination of the distributions in Figs. 4 and 5 suggests that there was less positional uncertainty, rather than more positional uncertainty, if a cue was presented.

A second hypothesis is that the effect of the cue is based on the relative strengths of activation of the representation of the initial location of the target and the representation of the cue at the time a participant responds. If the cue is presented after the target has appeared, then when a participant responds, the representation of the cue might be relatively more activated than is the representation of the initial location of the target, and so the cue would be more effective in modulating or overwriting information in the representation of the initial location of the target. If the cue is presented before the target has appeared, then when a participant responds, the representation of the cue might be relatively less activated than is the representation of the initial location of the target, and so the cue would be less effective in modulating or overwriting information in the representation of the initial location of the target. Thus, when a cue was presented, participants' responses in Experiments 1 and 2 might have relied more on memory for the target than on memory for the cue, whereas participants' responses in Experiments 3 and 4 might have relied more on memory for the cue than on memory for the target. In an extreme version of this hypothesis, participants in Experiments 3 and 4 might have ignored the target, but this is not consistent with the lack of differences in the overall probabilities of same responses as a function of cue presentation in Experiments 1 and 3 and, as was noted earlier, is not consistent with similarities of responses across Experiments 3 and 4 or with the occurrence of onset repulsion if cues were presented in Experiments 1 and 2 .

The decrease in the onset repulsion effect if a spatial cue that indicated the initial location of the target was presented in the present experiments is similar to the decrease in the Fröhlich effect if a spatial cue that indicated the initial location of the target was presented in Müsseler and Aschersleben (1998). However, the potential mechanism for the decrease in the Fröhlich effect proposed by Müsseler and Aschersleben does not seem applicable to the decrease in the onset repulsion effect: A facilitation of perceptual awareness that would allow perception of an earlier portion of the target trajectory could not account for an apparent perception of the target in a location (shifted from the actual initial location in the direction opposite to target motion) that the target never occupied. The decrease in the onset repulsion effect if a spatial cue that indicated the initial location of the target was presented in the present experi- ments is similar to the decrease in representational momentum if a spatial cue that indicated the final location of the target was presented in Hubbard et al. (2009). Spatial cuing can decrease the onset repulsion effect, and this is consistent with previous findings that spatial cuing can decrease the Fröhlich effect and can decrease representational momentum. ${ }^{4}$ These different types of displacement of represented spatial location involve processes that appear to be (at least partially) automatic, and explicit allocation of attention to the relevant part of the target trajectory appears to counteract at least some of the automatic displacement.

It should be noted that an onset repulsion effect was observed in the present experiments even though the targets exhibited implied motion. Thornton (2002) reported an onset repulsion effect with a form of implied motion that involved only the initial location and the final location of the target (but see Kerzel \& Gegenfurtner, 2004), but most subsequent studies of the onset repulsion effect involved presentation of smooth motion. The present experiments demonstrated that an onset repulsion effect can result from a sustained implied motion that used more than two inducing stimuli (see also Hubbard \& Courtney, 2008). It should also be noted that an onset repulsion effect was observed in the present experiments even though the response measure involved probe judgments of relative location. Previous reports of the onset repulsion effect involved pointing responses (positioning a cursor via movements of a computer mouse, Hubbard \& Motes, 2002, and Kerzel, 2002; reaching with the hand to a touchscreen, Kerzel \& Gegenfurtner, 2004), and Kerzel and Gegenfurtner (2004) suggested that there was a motor component to the onset repulsion effect. However, obtaining robust onset repulsion with probe judgments suggests that a motor component is not a necessary element of onset repulsion. Therefore, in addition to examining the effects of spatial cuing, the present experiments also contribute to the literature on the onset repulsion effect by revealing that the onset repulsion effect can occur when stimulus presentation involves implied motion and the response measure involves probe judgments of relative location.

The onset repulsion effect in memory for the initial location of a target was decreased or eliminated if a spatial

\footnotetext{
${ }^{4}$ Several studies showed a reduction in another type of spatial bias, the flash-lag effect (i.e., a briefly presented stationary object aligned with a moving object appears to be [i.e., appears to lag] behind the moving object; Nijhawan, 2002), when more attention is allocated to the location of the flash (e.g., Baldo, Kihara, Namba, \& Klein, 2002; Namba \& Baldo, 2004; Sarich, Chappell, \& Burgess, 2007; Shiori, Yamamoto, Oshida, Matsubara, \& Yaguchi, 2010; but see Khurana, Watanabe, \& Nijhawan, 2000). However, the flash-lag effect involves additional elements (e.g., judgment of the relative positions of two stimuli, rather than judgment of the absolute position of one stimulus), and so any relationship between attention in the onset repulsion effect and attention in the flash-lag effect is more speculative.
} 
cue that indicated the initial location of the target was presented before the target appeared, during target motion, or after the target vanished. The decrease in or elimination of the onset repulsion effect if a cue was presented is consistent with the positional uncertainty hypothesis, which suggests that the onset repulsion effect results from uncertainty regarding the initial location of the target. However, not all of the findings reported here were completely consistent with the positional uncertainty hypothesis, and so it is likely that positional uncertainty contributes to or enables the onset repulsion effect but is not the only mechanism or cause of the onset repulsion effect. The onset repulsion effect can be decreased by an increase in the allocation of attention to the initial location of the target, and this is not consistent with a hypothesis that attention is necessary to generate or maintain displacement. The effects of an increase in the allocation of attention to the initial location of the target on the onset repulsion effect in the present experiments are similar to the effects of an increase in the allocation of attention to the initial location or final location of the target on the Fröhlich effect or on representational momentum, respectively, in previous experiments, and so there appear to be interesting similarities in the influences of attention on the representation of target trajectory for different portions of that trajectory.

\section{References}

Actis-Grosso, R., \& Stucchi, N. (2003). Shifting the start: Backward mislocation of the initial position of a motion. Journal of Experimental Psychology: Human Perception and Performance, 29, 675-691.

Baldo, M. V. C., Kihara, A. H., Namba, J., \& Klein, S. A. (2002). Evidence for an attentional component of the perceptual misalignment between moving and flashing stimuli. Perception, $31,17-30$.

Hayes, A. E., \& Freyd, J. J. (2002). Representational momentum when attention is divided. Visual Cognition, 9, 8-27.

Hubbard, T. L. (2005). Representational momentum and related displacements in spatial memory: A review of the findings. Psychonomic Bulletin \& Review, 12, 822-851.

Hubbard, T. L., \& Bharucha, J. J. (1988). Judged displacement in apparent vertical and horizontal motion. Perception \& Psychophysics, 44, 211-221.

Hubbard, T. L., \& Courtney, J. R. (2008). The onset repulsion effect and motion induced mislocalization of a stationary object. Perception, 37, 1386-1398.

Hubbard, T. L., \& Motes, M. A. (2002). Does representational momentum reflect a distortion of the length or the endpoint of a trajectory? Cognition, 82, B89-B99.

Hubbard, T. L., \& Motes, M. A. (2005). An effect of context on whether memory for initial position exhibits a Fröhlich effect or an onset repulsion effect. Quarterly Journal of Experimental Psychology, 58A, 961-979.

Hubbard, T. L., Kumar, A. M., \& Carp, C. L. (2009). Effects of spatial cueing on representational momentum. Journal of Experimental Psychology: Learning, Memory, and Cognition, 35, 666-677.
Johnston, H. M., \& Jones, M. R. (2006). Higher order pattern structure influences auditory representational momentum. Journal of Experimental Psychology: Human Perception and Performance, $32,2-17$.

Kerzel, D. (2000). Eye movements and visible persistence explain the mislocalization of the final position of a moving target. Vision Research, 40, 3703-3715.

Kerzel, D. (2002). Different localization of motion onset with pointing and relative judgements. Experimental Brain Research, 145, $340-350$.

Kerzel, D. (2003). Attention maintains mental extrapolation of target position: Irrelevant distractors eliminate forward displacement after implied motion. Cognition, 88, 109-131.

Kerzel, D. (2010). The Fröhlich effect: Past and present. In R. Nijhawan \& B. Khurana (Eds.), Space and time in perception and action (pp. 321-337). Cambridge: Cambridge University Press.

Kerzel, D., \& Gegenfurtner, K. R. (2004). Spatial distortions and processing latencies in the onset repulsion and Fröhlich effects. Vision Research, 44, 577-590.

Khurana, B., Watanabe, K., \& Nijhawan, R. (2000). The role of attention in motion extrapolation: Are moving objects "corrected" or flashed objects attentionally delayed? Perception, 29, 675-692.

Kirschfeld, K., \& Kammer, T. (1999). The Fröhlich effect: A consequence of the interaction of visual focal attention and metacontrast. Vision Research, 39, 3702-3709.

Munger, M. P., Solberg, J. L., Horrocks, K. K., \& Preston, A. S. (1999). Representational momentum for rotations in depth: Effects of shading and axis. Journal of Experimental Psychology: Learning, Memory, and Cognition, 25, 157-171.

Müsseler, J., \& Aschersleben, G. (1998). Localizing the first position of a moving stimulus: The Fröhlich effect and an attentionshifting explanation. Perception \& Psychophysics, 60, 683-695.

Müsseler, J., \& Kerzel, D. (2004). The trial context determines adjusted localization of stimuli: Reconciling the Fröhlich and onset repulsion effects. Vision Research, 44, 2201-2206.

Müsseler, J., Stork, S., \& Kerzel, D. (2008). Localizing the onset of moving stimuli by pointing or relative judgment: Variations in the size of the Fröhlich effect. Vision Research, 48, 611-617.

Namba, J., \& Baldo, M. V. C. (2004). The modulation of the flash-lag effect by voluntary attention. Perception, 33, 621-631.

Nijhawan, R. (2002). Neural delays, visual motion and the flash-lag effect. Trends in Cognitive Sciences, 6, 387-393.

Posner, M. I., Nissen, M. J., \& Ogden, W. C. (1977). Attended and unattended processing modes: The role of set for spatial location. In H. L. Pick \& I. J. Saltzman (Eds.), Modes of perceiving and processing information (pp. 137-157). Hillsdale, NJ: Erlbaum.

Sarich, D., Chappell, M., \& Burgess, C. (2007). Dividing attention in the flash-lag illusion. Vision Research, 47, 544-547.

Shiori, S., Yamamoto, K., Oshida, H., Matsubara, K., \& Yaguchi, H. (2010). Measuring attention using the flash-lag effect. Journal of Vision, 10(10, Art. 10), 1-13.

Teramoto, W., Hidaka, S., Gyoba, J., \& Suzuki, Y. (2010). Auditory temporal cues can modulate visual representational momentum. Attention, Perception, \& Psychophysics, 72, 2215-2226.

Thornton, I. M. (2002). The onset repulsion effect. Spatial Vision, 15, 219-243.

Tsal, Y., \& Bareket, T. (1999). Effects of attention on localization of stimuli in the visual field. Psychonomic Bulletin \& Review, 6, 292-296.

Tsal, Y., \& Bareket, T. (2005). Localization judgments under various levels of attention. Psychonomic Bulletin \& Review, 12, 559-566.

Verfaillie, K., \& d'Ydewalle, G. (1991). Representational momentum and event course anticipation in the perception of implied periodical motions. Journal of Experimental Psychology: Learning, Memory, and Cognition, 17, 302-313. 